\title{
OPTIMIZATION AND DESIGN OF A CABLE DRIVEN UPPER ARM EXOSKELETON
}

\author{
Sunil K. Agrawal ${ }^{1}$, Venketesh N. Dubey ${ }^{2}$, John J. Gangloff Jr. ${ }^{1}$, Elizabeth Brackbill ${ }^{1}$ and Vivek Sangwan ${ }^{1}$ \\ ${ }^{1}$ Mechanical Systems Laboratory, Department of Mechanical Engineering, University of Delaware, Newark, DE, 19716, USA \\ ${ }^{2}$ School of Design, Engineering and Computing, Bournemouth University, Fern Barrow, Poole, BH12 5BB, UK
}

\begin{abstract}
This paper presents the design of a wearable upper arm exoskeleton that can be used to assist and train arm movements of stroke survivors or subjects with weak musculature. In the last ten years, a number of upper-arm training devices have emerged. However, due to their size and weight, their use is restricted to clinics and research laboratories. Our proposed wearable exoskeleton builds upon our extensive research experience in wire driven manipulators and design of rehabilitative systems. The exoskeleton consists of three main parts: (i) an inverted U-shaped cuff that rests on the shoulder, (ii) a cuff on the upper arm, and (iii) a cuff on the forearm. Six motors, mounted on the shoulder cuff, drive the cuffs on the upper arm and forearm, using cables. In order to assess the performance of this exoskeleton, prior to use on humans, a laboratory test-bed has been developed where this exoskeleton is mounted on a model skeleton, instrumented with sensors to measure joint angles and transmitted forces to the shoulder. This paper describes design details of the exoskeleton and addresses the key issue of parameter optimization to achieve useful workspace based on kinematic and kinetic models.
\end{abstract}

Keywords: Arm Exoskeleton, Cable Driven, Rehabilitative Device, Optimization, Orthotic systems

\section{INTRODUCTION}

A vast number of people are affected by arm conditions due to degeneration of muscles that result in profound muscle weakness or impaired motor control such as, people suffering from muscular diseases like Spinal Muscular Atrophy (SMA), Duchenne Muscular Dystrophy (DMD) and Limb Girdle. They have problems in lifting the arms against gravity [1].
Consequently, they are not able to perform various activities of daily living (ADL) independently. External mechanical support can provide assistance to movement of the arm when adequate motor control is lacking, this would greatly improve the handling capability in people with functional deficits. However, none of the existing exoskeleton designs have the potential to be lightweight and easy to be worn continuously. Some wellknown designs to evaluate and treat upper extremity functions for post-stroke rehabilitation of patients employing robotic technology are ARM [2], MIME [3] and MIT-MANUS [4]. However, none of these were meant to be portable support devices. Since then, there has been continuous surge of interest in developing upper limb exoskeleton for training and assistance. In the recent years, new developments of such devices are keenly pursued. A 7-dof powered exoskeleton was developed as a therapeutic and diagnostics device for human power amplifications [5]. However, this device has a rigid mechanical structure which can only lend itself for training purposes. Wearability and continuous use as a support device is not possible; most existing/recent exoskeletons fall under this category [6-9]. To make exoskeletons lighter and wearable, some clever designs are reported with cable based systems, however, the final system still appeared to be bulky with actuators and controllers $[10,11]$; other designs were limited to modeling and simulation $[12,13]$.

The challenging problems in exoskeleton design are of weight, power, size and functionality to be achieved without overburdening either the components or the user. Existing electric powered elbows can attain about $12.2 \mathrm{Nm}$ of lift by the elbow's own motor mechanism at speeds of about $2 \mathrm{rad} / \mathrm{s} \mathrm{[14],}$ however, if such devices are to be externally powered then it should be able to run for a whole day from the same power source without needing to be replaced or recharged and should 
be contained within the body to be portable. Currently, such a versatile power source is not available which means that the mechanical components need to be designed in such a way that they require least torque and can reach every configuration. This paper presents design of a wearable, cable-driven exoskeleton that can be worn and operated in a natural way and considers parameters optimization of the exoskeleton to maximize the operating workspace based on its kinematic model.

\section{DESIGN DETAILS AND EXOSKELETON KINEMATICS}

Our initial design based on these criteria is developed in Solidworks $^{\text {TM }}$ (Fig. 1). The design is inspired by the actuation mechanism of the natural arm to make it light weight and wearable. However, it not intended to provide full functional capability to the natural arm, since the design has a limited number of actuators. The cable driven exoskeleton, in the current laboratory set up, is fitted to a skeleton arm. The exoskeleton has two cuffs for the upper and forearm and an inverted U-shaped shoulder cuff over the scapula (shoulder blade) holding the motors. The cuffs have a channel section which holds circular tubes that can be inflated/deflated for sufficient grip on the contact parts. The cuffs also have attachment points for the cables. The exoskeleton uses 6 small motors, 4 for the shoulder joint and 2 for the elbow joint; a minimum of $n+1$ cables are required to achieve $n$-degrees of freedom [15]. The shown skeleton emulates the motion of human arm with three rotational joints at the shoulder and a revolute joint at the elbow. This has been achieved by especially designing the shoulder joint which provides the useful range of anatomical motions. The skeleton has a force torque sensor at the shoulder joint and encoder at each rotational axis to obtain force and motion data during experimentation.

An experimental rig was developed based on the above model as shown in Fig. 2 which uses a plastic skeleton interfaced with these sensors. As seen, this uses a total of three cuffs - first cuff sits on the shoulder, one cuff wraps around the upper arm, and the third one goes on to the forearm. The arm motion is controlled by cables attached to the cuffs that are driven by motors mounted to top of the shoulder cuff. The prototype has been machined from aluminum. One of the main objectives of the experimental exoskeleton is to track the angular position and velocity of the arm while executing motion sequence together with the force and moment components at the shoulder joint. The natural ball-and-socket joint of shoulder within the skeleton does not have fixed axes of rotation. This will present difficulty in accurately tracking each axis of rotations since encoders cannot be mounted independently. This was achieved by designing a new shoulder joint which has three distinctive axes of rotation corresponding to the natural ball-and-socket joint.

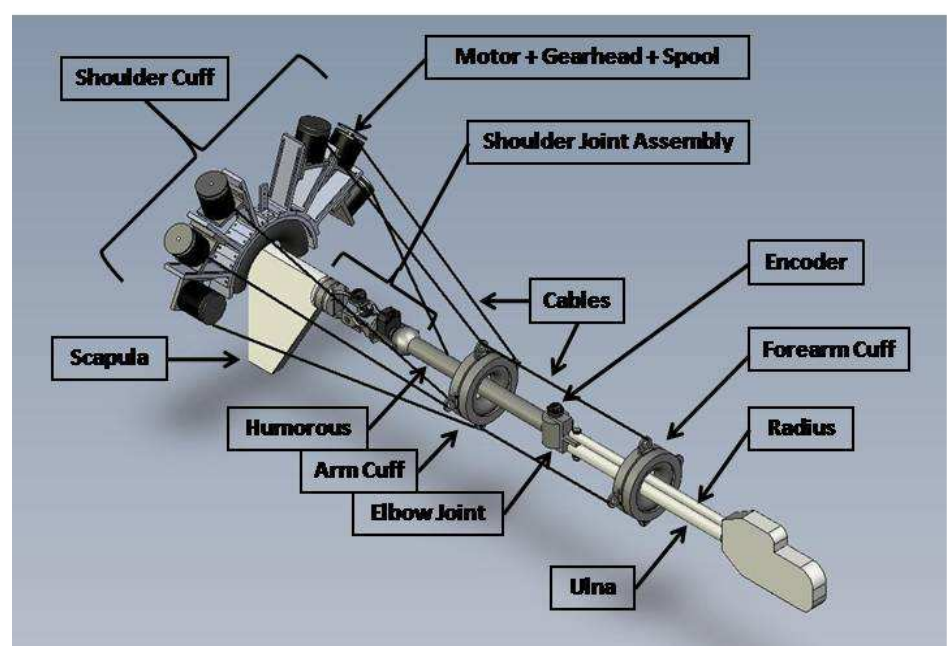

Figure 1. Model of the exoskeleton on a skeleton arm

The novel features of our exoskeleton design are: (i) it is modular and can be easily adjusted with optimized parameters (ii) it is light weight, cable driven, self-contained, and portable and (iii) it can be optimized for global tasks as well as specific tasks. On the flip side, it is difficult to achieve positive tension in all the cables over its workspace using a minimum number of actuators. However, once satisfactory performance of the exoskeleton is achieved in terms of range of motion and reaction forces at the shoulder joint, it can be customized much like a wearable jacket which can be tested on human arm and clinically evaluated for regular use.

Due to the very nature of cable driven systems, which can only apply pulling forces, it is necessary to keep the design modular to accommodate adjustments in motor mounting and cable attachment points so that tension in cables can remain positive. In order to achieve this, the shoulder cuff design has been made with various tracks and groves to permit motors placement at different radial $(r)$ as well as angular positions $(\Psi)$ as shown in Fig.3. In addition, due to the size of motors and physical constraints of the design to accommodate six motors on the shoulder cuff, use of radial fins have been made which can hold motors on either sides of the plate. The motors can also be flipped by $90^{\circ}$ on the radial fins to further allow adjustments on the cable attachment points (as shown by the first motor on the left hand side in Fig. 3). Up to two motors can be placed on a fin, which provides flexibility in achieving optimized workspace for the arm. Flexibility in motor placement was paramount in shoulder cuff design since the motors could be placed at their ideal locations for different geometric and inertial parameters of the arm to maximize the workspace. The main question is how to optimize these parameters for maximizing the workspace, this requires kinematic/dynamic model of the exoskeleton to be developed. 


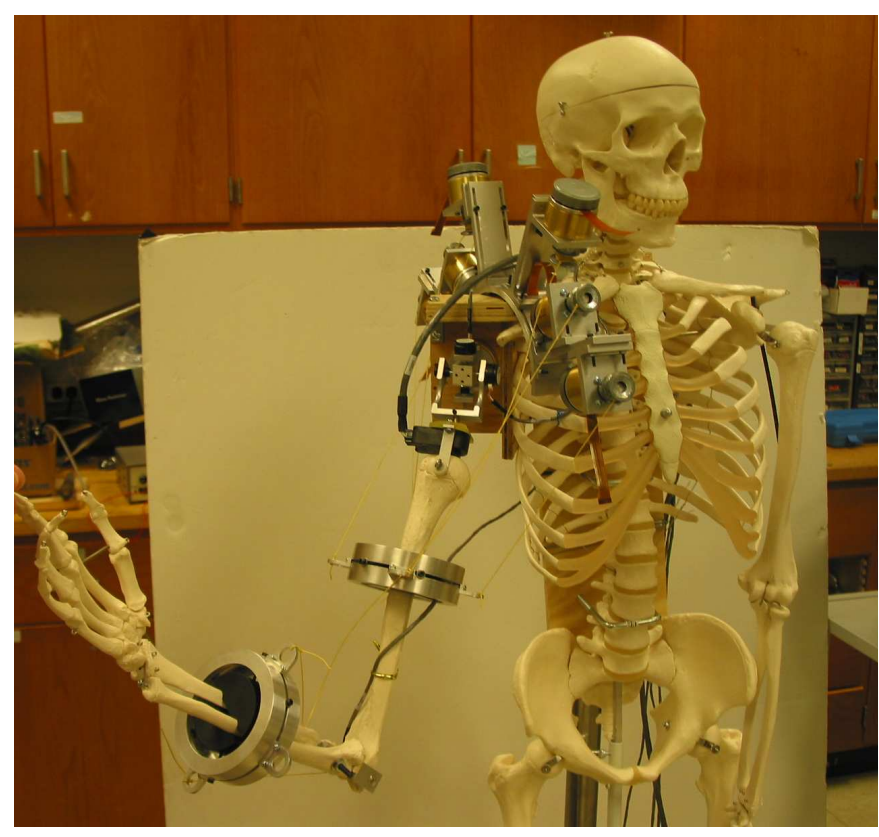

Figure 2. Exoskeleton fitted on a plastic arm with cable driven cuffs

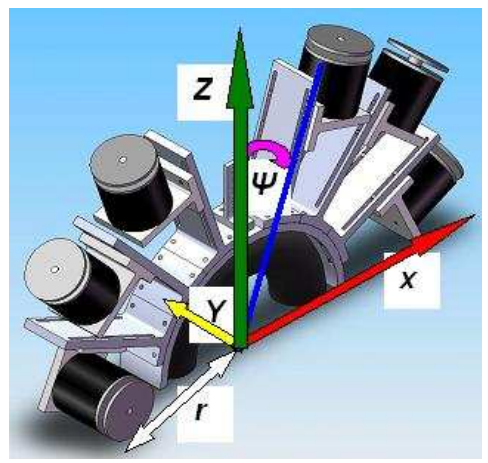

Figure 3. Shoulder cuff design with adjustable motor placement

The kinematic parameters of the exoskeleton arm are shown in Fig. 4 and the DH-parameters are listed in Table 1. The exoskeleton has four links and it provides 4 DOF (3 at the shoulder and 1 at the elbow). As seen in the figure, Body 1 is the cross bar holding the arm, Body 2 is the U-link connecting the cross bar to the arm, Body 3 and Body 4 are the upper and lower arms, respectively.

\begin{tabular}{|c|c|c|c|c|}
\hline Link/Parameters & $a_{i}$ & $\alpha_{i}$ & $d_{i}$ & $\theta_{i}$ \\
\hline 1 & 0 & 90 & 0 & $\theta_{1}$ \\
\hline 2 & 0 & 90 & 0 & $\theta_{2}$ \\
\hline 3 & 0 & 90 & $d_{3}$ & $\theta_{3}$ \\
\hline 4 & $a_{4}$ & 0 & 0 & $\theta_{4}$ \\
\hline
\end{tabular}

Table 1. DH-parameters of the exoskeleton arm

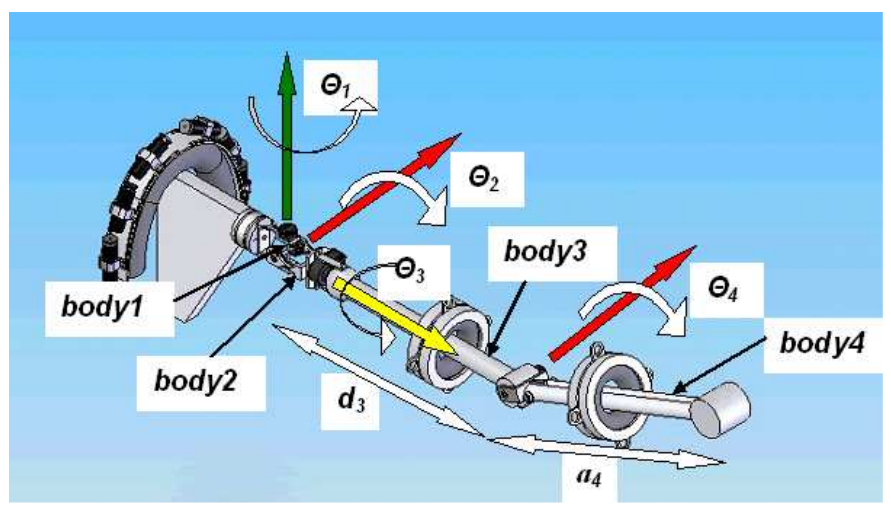

Figure 4. Parameters for kinematic modeling

\section{WORKSPACE OPTIMIZATION}

In order to assist the motion of the arm, it needs to be actuated through cables connected to the cuffs. The design forms a cable driven parallel mechanism for which workspace characterization is essential $[16,17]$. Ideally, the exoskeleton should be able to perform all reaching tasks under gravity and inertial loads of the arm. However, due to unilateral property of cables to only pull and for the given architecture, it can only achieve a subset of all reachable locations. In other words, the exoskeleton will have a static and dynamic workspace smaller than the reachable workspace. The dynamic model of the exoskeleton was developed using Lagrangian formulation:

$$
D(q) \ddot{q}+C(q, \dot{q}) \dot{q}+g(q)=J(q)^{T} T(t)
$$

where $q=\left(\theta_{1}, \theta_{2}, \theta_{3}, \theta_{4}\right)^{T}$ are the generalized coordinates, $\mathrm{D}(\mathrm{q})$ is the $(4 \times 4)$ inertia matrix, $C(q, \dot{q})$ is the vector of nonlinear centripetal terms, $g(q)$ is the vector of gravity terms, $J(q)$ is the Jacobian relating the cable attachment points, and $T(t)$ is a six dimensional cable tension vector. Due to complexity of the dynamical model, we only provide a functional form of the equation here. Detailed models were developed in Maple ${ }^{\mathrm{TM}}$ and MATLAB ${ }^{\mathrm{TM}}$ and are being used in MATLAB for numerical simulation with the form of Eq. (1).

This model was used for static workspace evaluation by setting $\dot{q}=0, \ddot{q}=0$. In the first step, this model was used for shoulder workspace evaluation. Parameter optimization was performed to see if useful workspace exists, i.e., the tension in the cable remains positive for the range of motion within the useful workspace. Later, this technique was extended for whole arm parameter optimization. The above equations, in statics, can be written in the general form,

$$
A T=B,
$$

Where,

$$
\begin{aligned}
& A=\left[\begin{array}{lll}
a_{1} & a_{2} \ldots & a_{6}
\end{array}\right] \in R^{4 \times 6} \quad \text { Here, } A=J(q)^{T} \text { and } \\
& B=g(q) . \\
& B=\left[V_{1}, V_{2} \ldots V_{4}\right]^{T} \in R^{4 \times 1} \text { is the vector of gravity terms }
\end{aligned}
$$


$T=\left[\begin{array}{lll}T_{1} & T_{2} \ldots . & T_{6}\end{array}\right]^{T} \in R^{6 \times l}$ is the cable tension vector and

The solution for cable tension is given by:

$$
T=\bar{T} B+N(A) m,
$$

where, $\bar{T}=A^{T}\left(A A^{T}\right)^{-1}$ is the pseudoinverse of matrix $A$, $N(A)$ is the null space of matrix $A$ and $m$ modulates tension in cables [18].

To simplify the workspace computation of the whole arm, Eq.

(2) is split into shoulder and elbow matrices as shown in Eq.

(4). The symbol ' $U$ ' is used represent the addition of useful elements of two unequal vectors. The elbow degree-of-freedom represented by Eq. (5), uses the last component equation of (2). Initially, positive tension is ensured in the elbow cables using Eq. (5), this gives elbow cable tension $\left(T_{5}, T_{6}\right)$. Then, Eq. (6) is used to ensure positive tension in the cables for the shoulder joint, as shown in Eq. (7). If positive tension is not possible for the elbow cables, it records this as an infeasible arm position and the program moves to a new test point. The whole arm optimization process is shown in the flow chart, Fig. 5. This technique has been used throughout to characterize the workspace and for exoskeleton parameter optimization.

$$
A_{S} T_{S} \cup A_{E} T_{E}=B
$$

Where,

$$
\begin{aligned}
A_{S} \in R^{3 \times 4}, T_{S}\left(T_{1}, \ldots, T_{4}\right) \in R^{4 \times 1}, \\
A_{E} \in R^{4 \times 2}, T_{E}\left(T_{5}, T_{6}\right) \in R^{2 \times 1} \\
A(4,5) T_{5}+A(4,6) T_{6}=B(4) \\
A_{S} T_{S}=\left(B-A_{E} T_{E}\right)=B_{s} \\
T_{S}=\bar{T}_{S} B_{S}+N\left(A_{S}\right) m
\end{aligned}
$$

where $\bar{T}_{S}$ is pseudoinverse of $A_{S}$ and

$$
B_{S}=\left(B-A_{E} T_{E}\right) \in R^{3 \times 1}
$$

\section{(A) SHOULDER PARAMETER OPTIMIZATION}

For the shoulder joint alone, Eq. (2) has a smaller size, i.e., $A$ is a $(3 \times 4)$ matrix, $B$ is a $(3 \times 1)$ vector and $T$ is a $(4 \times 1)$ vector. Since $A$ is nonlinear containing many trigonometric functions, it is hard to get analytical solution for positive cable tension using the pseudoinverse. Hence, this is done numerically by modulating the null-space parameter $m$. The feasible solution of $m$ is characterized by a convex region bounded by $n$ linear inequality on the parameter $m$. With one extra cable, for a 3-dof joint, the four linear inequalities in $m$ are given by Eq. (8).

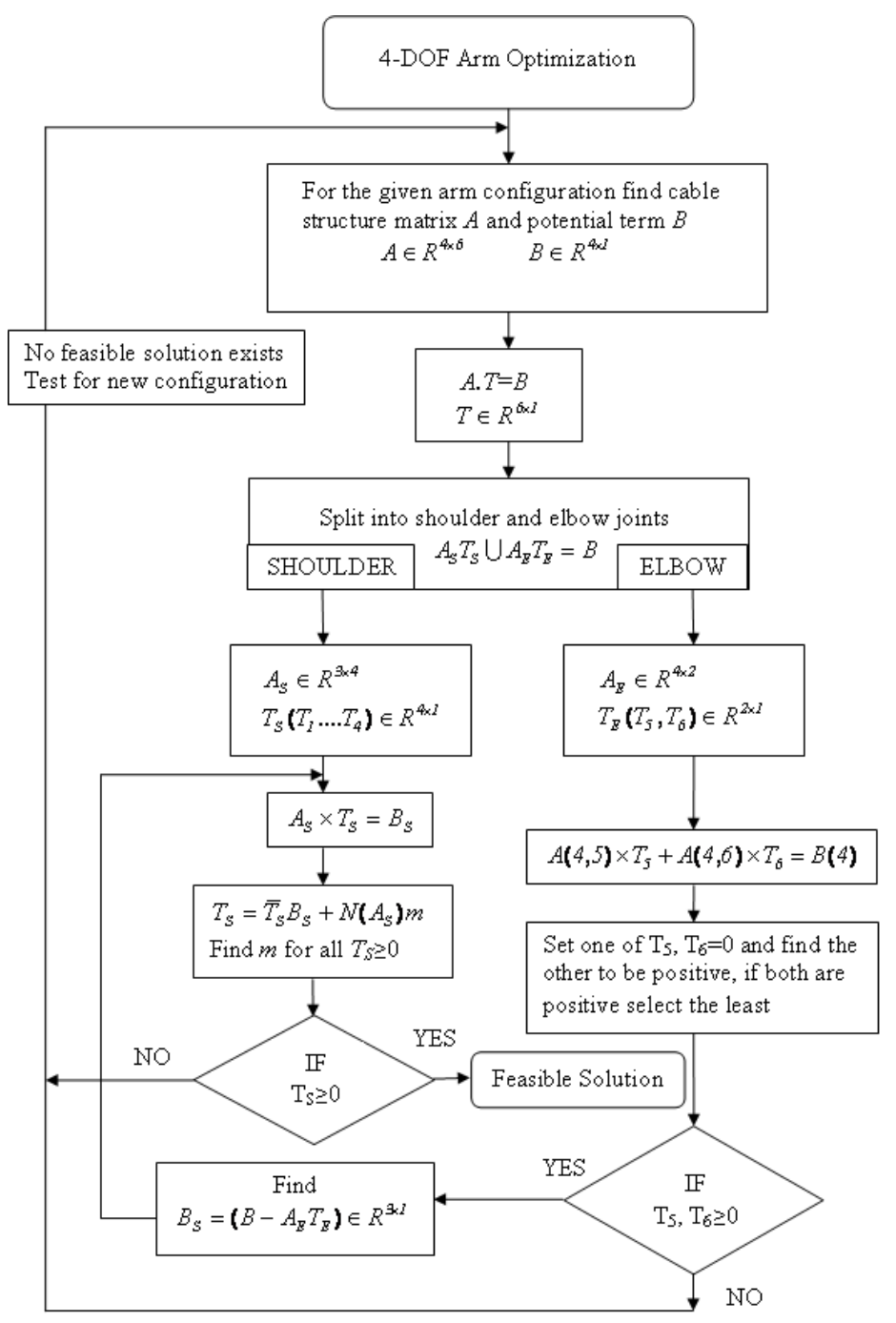

Figure 5. Flow chart of optimization process, the whole arm is split into shoulder and elbow joint cables, initially positive tension is ensured in the elbow cables this results in coupling at the shoulder where positive tension conditions are then satisfied.

$$
\left[\begin{array}{l}
\bar{T}_{1} \\
\bar{T}_{2} \\
\overline{T_{3}} \\
\bar{T}_{4}
\end{array}\right]+\left[\begin{array}{l}
n_{11} \\
n_{21} \\
n_{31} \\
n_{41}
\end{array}\right] m \geq 0
$$

The feasible region $F_{A}$ of $m$ is described by the common interval bounded by four linear inequalities as shown in Figure 6. Here $p_{i}$ is the solution point when each component of Eq. (8) is an equality. If $F_{A}$ is empty, the tension constraints cannot be met. 


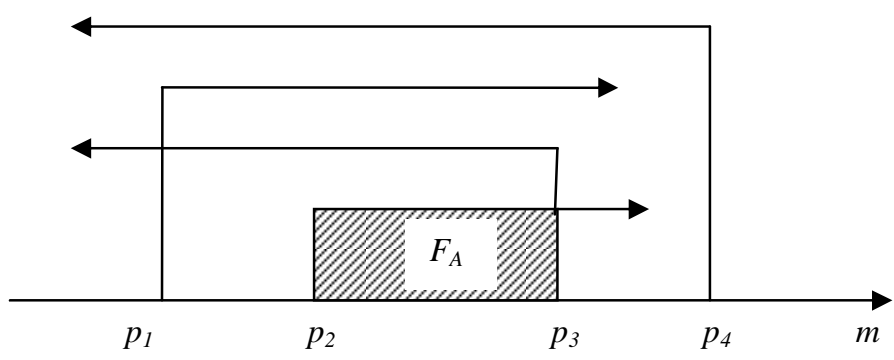

Figure 6. A sketch of a feasible region for $m$ for a 3-dof shoulder joint driven by four cables

As discussed earlier, there are many variables in the shoulder cuff design that may be considered for maximizing the workspace keeping the cable tension positive for the range of arm motion. However, some of these parameters cannot be changed due to physical limitations of the design or due to the constraints in operating range. Most problems of constrained nonlinear multivariable function are best solved by evolutionary methods, whereby, a problem with a smaller number of independent variables is solved first. Optimization problems also benefit from good starting guesses, simpler cost functions and less stringent termination criteria to reduce the computation time- this improves the execution efficiency and can help locate better local minima. With this in mind, only the angular spacing between the attachment points on the shoulder and upper arm cuffs were initially considered for optimization. Fig. 7 shows the initial setting of the attachment points with the reference axis for each cuff. As can be seen, there are a large number of variables responsible for performance of the exoskeleton e.g. angular attachment angles on the cuffs $\left(\Psi_{1}\right.$, $\left.\Psi_{2}\right)$, radial positions $\left(r_{0}, r_{1}\right)$ and axial positions along $\left(y_{0}\right.$ axis) the arm. The following are the initial settings for the exoskeleton range of operation.

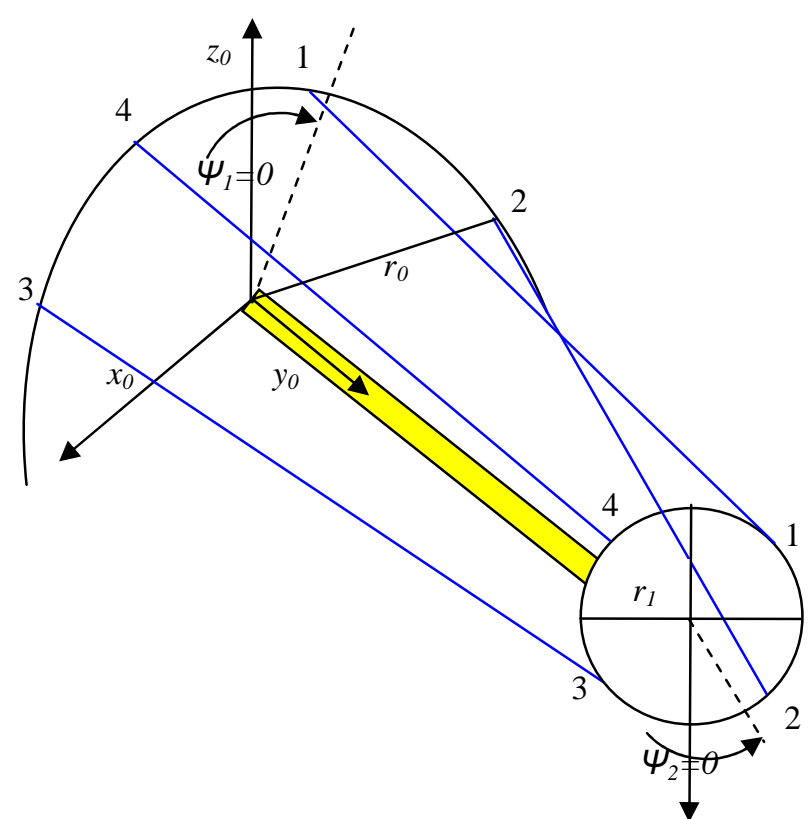

Figure 7. Cable attachment points and optimization parameters as shown with respect to the local coordinate frame and the sense of rotation on the plane of cuffs.
Range of shoulder joint angles (refer to Fig. 4):

$-80<\theta_{1}<80,10<\theta_{2}<170,-60<\theta_{3}<60$

$\Psi_{1}$ is the angular orientation (in degrees) of the cable attachment points $(1,2,3,4)$ as shown on the shoulder cuff. This is with respect to the local coordinate frame and the sense of rotation starting at an angle $\left(\Psi_{1}=0\right)$ on the plane containing the shoulder cuff. These are given by $\Psi_{1}=[30 ; 60 ;-60 ;-30]$. $\Psi_{2}$ is the corresponding angular orientation of the cable attachment points on the upper arm cuff given by $\Psi_{2}=[135 ; 45$; $-45 ;-135]$. The cuff's radii are $r_{0}=0.1 \mathrm{~m}$ and $r_{l}=0.05 \mathrm{~m}$. The shoulder cuff is placed at the reference plane (i.e. no shift along $y_{0}$ axis) and upper arm cuff is located at $0.15 \mathrm{~m}$ along the arm. With these settings, the model calculates the cable tension and, if negative, attempts to make it positive using the modulator $m$ in Eq. (8). Figure 8 shows the qualitative workspace for a unit arm connected to the shoulder joint, higher the color map order, better is the workspace feasibility. The workspace is hemispherical about the shoulder joint (at the origin of the coordinate frame), clearly the workspace is small and discontinuous at this setting.

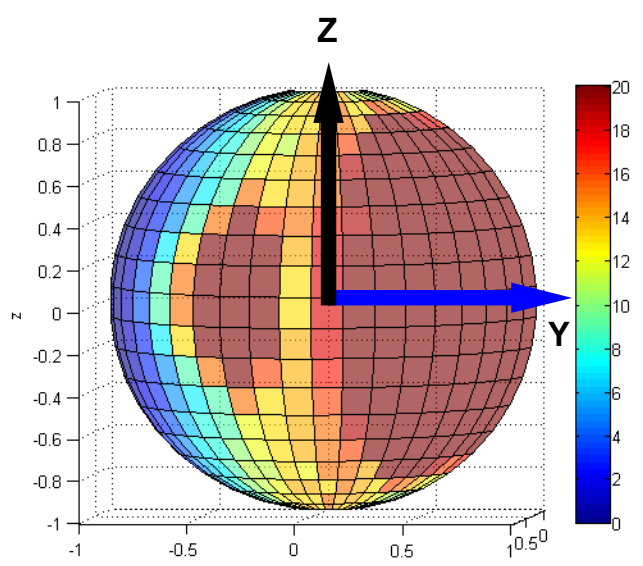

Fig. 8 Feasible workspace (qualitative) at initial setting

In order to maximize the feasible workspace, the model formulation is subjected to the MATLAB fmincon optimization function; this is designed to solve nonlinear programming problems such as computation of continuous trajectories for control [18]. The lower and upper bounds (LB \& UB) of a sample attachment point on the shoulder cuff is shown in Figure 9 for optimization. Only angular positions of cable attachment points were considered here, this helps in fast convergence of optimization process as well as facilitates flexibility in physical assembly of components of the exoskeleton. The objective function is to minimize the number of infeasible points in each run.

$\boldsymbol{\Psi}_{1 \_L O W E R}=[15 ; 45 ;-75 ;-45]$
$\boldsymbol{\Psi}_{1 \_U P P E R}=[45 ; 75 ;-45 ;-15]$
$\boldsymbol{\Psi}_{2 \_L O W E R}=[90 ; 0 ;-90 ;-180]$
$\boldsymbol{\Psi}_{2 \_U P P E R}=[180 ; 90 ; 0 ;-90]$

$\Psi_{1 \_L O W E R}=[15 ; 45 ;-75 ;-45]$

$\Psi_{2}{ }_{L O W E R}=[90 ; 0 ;-90 ;-180]$ 


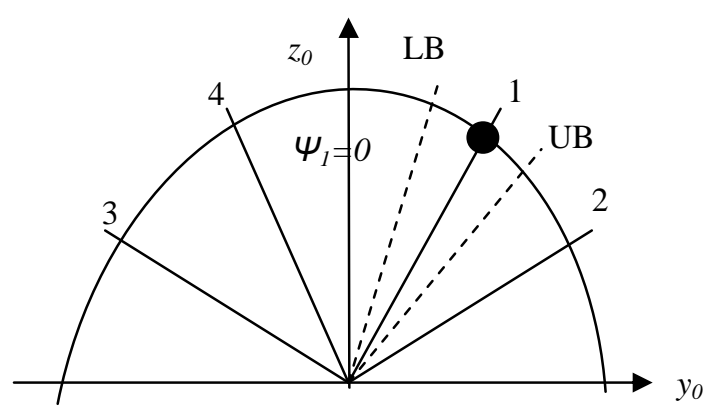

Figure 9. Upper and lower bound of an attachment point on the shoulder cuff

Figure 10 shows the optimized result for the above case which offers the following angles (rounded to the closest integer) for cable attachment points for the given joint angle range. It can be clearly seen that the workspace has significantly improved. Once the optimized solution is obtained, effect of change of radial position of the attachment points is evaluated. It has been found that it has marginal effect on the workspace, slightly improves with the increasing radius.

$\Psi_{1 \_O P T I M I Z E D}=[15 ; 75 ;-46 ;-15]$

$\Psi_{2}$ OPTIMIZED $=[158 ; 0 ;-15 ;-180]$

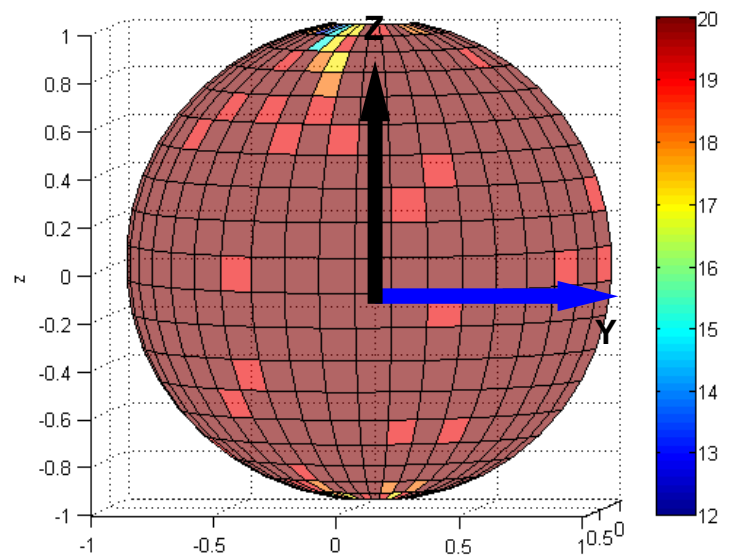

Fig. 10 Optimized workspace due to joint motion at the shoulder.

\section{(B) WHOLE ARM OPTIMIZATION}

Based on the formulation in Figure 5, optimization for the whole arm was carried out with the following initial settings. Range of joint angles on shoulder and elbow is as shown (refer to Fig. 4), this adequately covers range of motions to carry out most ADLs.

$-70<\theta_{1}<70,10<\theta_{2}<100,-20<\theta_{3}<20,-70<\theta_{4}<90$

Angular orientation (in degrees) of the cable attachment points on the shoulder cuff are, $\boldsymbol{\Psi}_{1}=[30 ; 60 ;-60 ;-30 ; 75$; -75 ]. Due to the operational requirement of the elbow joint, the plane containing the elbow cables must be perpendicular to the joint axis at all time. As a result, angular attachment of elbow cables on upper and lower arm are fixed and not subjected to optimization. This is given (with reference to the local frame) by $\Psi_{2 \text { ELBOW }}[90 ;-90]$ and $\boldsymbol{\Psi}_{3}=[180 ; 0]$. Therefore, angular orientation (in degrees) of the cable attachment points on the upper arm cuff are $\Psi_{2}=[135 ; 45$; $-45 ;-135]$. The cuff radius at shoulder, upper arm and forearm are $r_{0}=0.1 \mathrm{~m} ; r_{1}=0.05 \mathrm{~m} ; r_{2}=0.05 \mathrm{~m}$ respectively and the length of upper and forearm are $0.3 \mathrm{~m}$ and $0.25 \mathrm{~m}$. The shoulder cuff is placed at the reference plane and the upper and lower arm cuffs are located at $0.15 \mathrm{~m}$ along their respective arms from the preceding joint. Due to the design constraints, following are the lower and upper bound set on the orientation of the cable attachment points. Figure 11 shows the workspace of the exoskeleton at initial setting and the optimized workspace is shown in 12 .
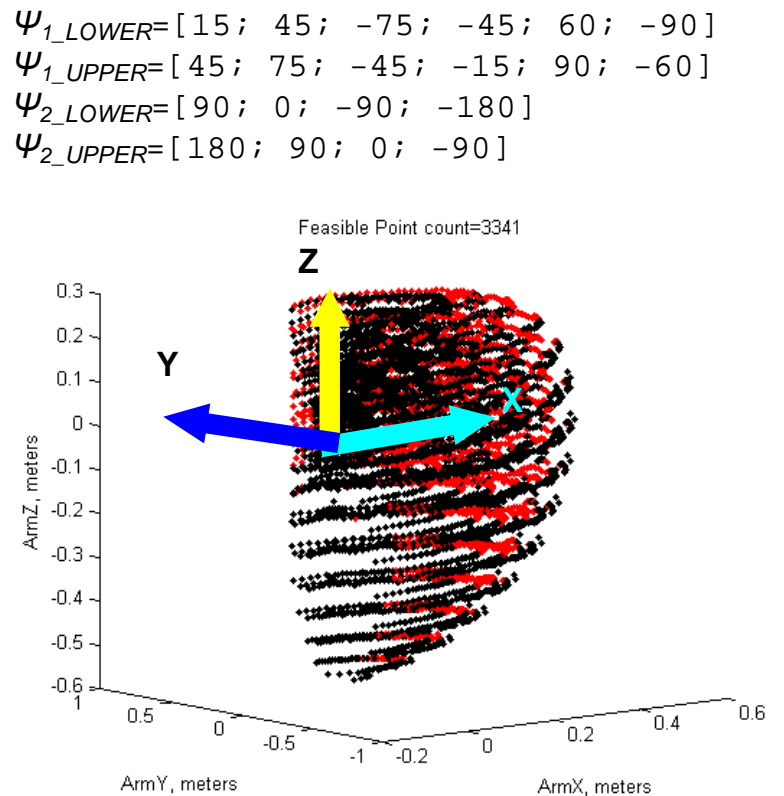

Figure 11. Exoskeleton workspace in initial setting.

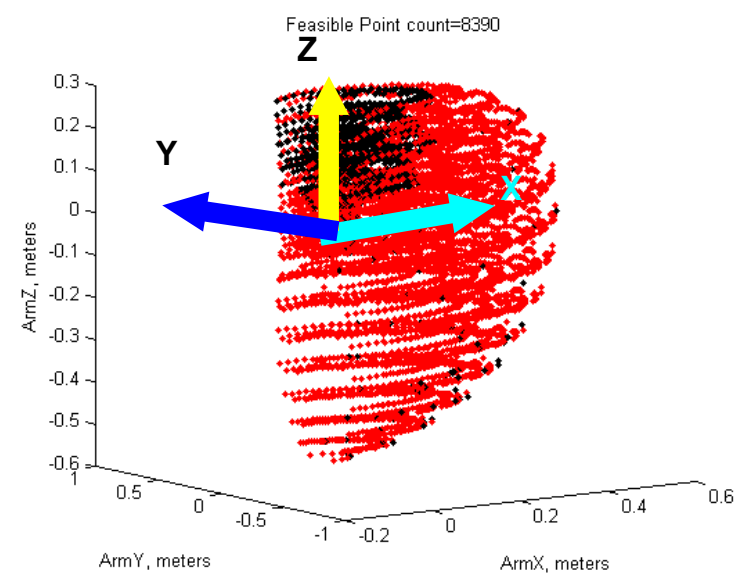

Figure 12. Exoskeleton workspace after optimization (redfeasible, black-infeasible points), 8390 feasible points achieved out of 10000 possible points compared to just 3341 in Fig. 11. 
As seen in Fig. 12, the workspace has improved significantly and the number of feasible points count increased from 3341 to 8390 (out of 10000). The center of the workspace is located at the origin of the coordinate frame. The infeasible points (black dots) are mostly seen at the top of shoulder joint, this is intuitively agreeable since the cable driven arm will have singular configuration at these locations. The optimization gives the following orientation angles (rounded to the closest integer) for cable attachment points for the given joint angle range.

$\boldsymbol{\psi}_{1 \_O P T I M I Z E D}=[15 ; 75 ;-75 ;-45 ; 90 ;-60]$

$\Psi_{2 \_O P T I M I Z E D}=[180 ; 0 ; 0 ;-180]$

The cable attachment points are shown graphically in Figure 13 from initial to an optimized condition. The top picture is the initial setting of the exoskeleton represented by the left hand line diagram - central red line is the extended arm and black lines represent the cuffs. The optimized configuration is shown in the right hand line diagram which can be reflected back into the original model for the intended application. The effect of change of radial position of the cable attachment points is further studied on the optimization result and contrary to the shoulder optimization it has been found that the workspace improves with decreasing radius.

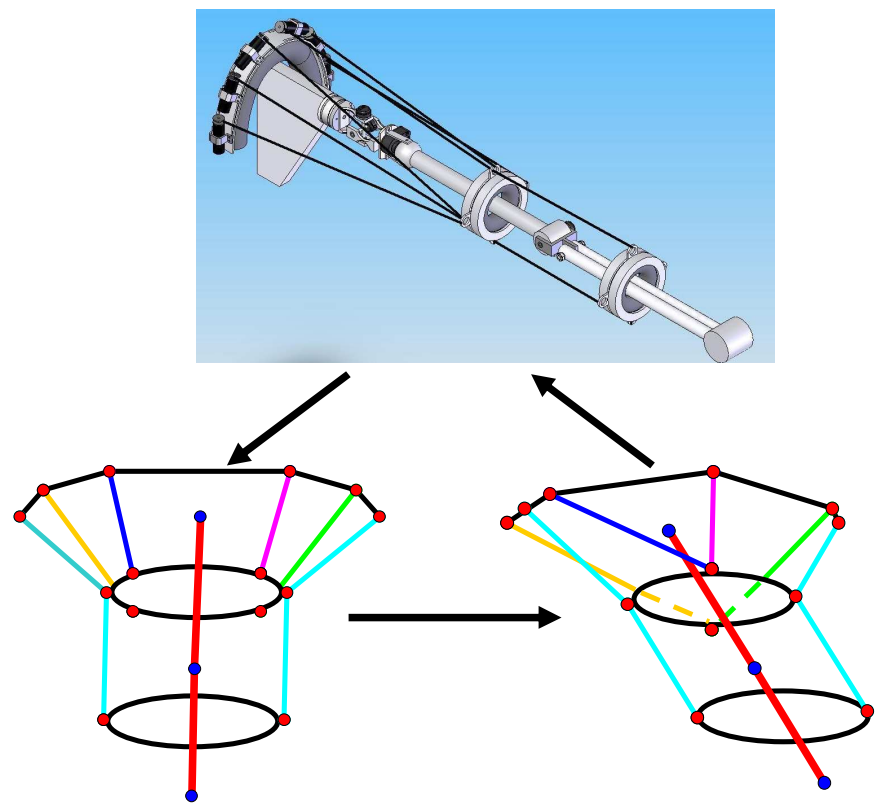

Figure 13. Optimization results for cable attachment points from initial to final configuration, the top plate shows the model in initial configuration represented by the left hand line diagram. The optimized arrangement is shown in the right hand line diagram going back to the new model setting-central (red) line is the arm in extended position and the outlines (black) represent the cuffs.

\section{CONCLUSIONS}

In this paper, optimization and design of a cable driven upper arm exoskeleton were presented. Our design is motivated from tendon-based natural arm by using a cable driven parallel mechanism. However, cable based parallel manipulators require additional motors for the same number of degrees-offreedom, since cables can only transmit power in tension. This motivates optimizing the design parameters to maximize the workspace with a limited number of actuators. It has been shown that optimization technique used in this paper significantly improved the workspace. Initially, the optimization was applied to the shoulder joints. Subsequently, whole arm optimization was carried out based on this technique. This was performed in two steps, first evaluating the elbow joint and then bringing the coupling of the elbow cables to the shoulder joints. One important difference noticed in these two optimizations is that in the case of the shoulder joint, the workspace improves by increasing the cuff radius, whereas, for the whole arm workspace improves with decreasing radius. This behavior is consistent with the arm where many tendons connect to a small region around the scapula, while the arm is still able to reach a range of locations. However, with limited number of actuators, the designed exoskeleton cannot be functionally compared to a natural arm, which has a large number of tendon-muscles combined. The optimization technique was further applied to specific cases of activities of daily living and it was found that the workspace can be further optimized for a specific purpose than a general optimization for the full motion range. This fits in very well with our modular design which can be easily tailored and optimized for specific applications. The presented design offers a practical proposition for training and assistance utilizing minimum of resources. Further to validate the exoskeleton compliance with anatomical arm movement and assess the load bearing capability on human subjects it is currently being evaluated with a sensor interface. The paper has shown that a lightweight wearable exoskeleton can be realized on a cable-driven manipulator that can be conveniently used for training and assistance.

\section{ACKNOWLEDGEMENT}

The support of second author was provided by the Global Research Award from the Royal Academy of Engineering of England and Bournemouth University (UK). The research was also supported by WCU (World Class University) program through the Korea Science and Engineering Foundation funded by the Ministry of Education, Science and Technology (No. R32-2008-000-10022-0).

\section{REFERENCES}

[1] Lucieer, P. and Herder, J. L. "Design of an adjustable compensation mechanism for use in a passive arm support", ASME International Design Engineering Technical Conference, DETC2005-85442, Long Beach, California (USA), Sept. 24-28, 2005.

[2] Reinkensmeyer, D. J., Hogan, N. et al. "Rehabilitators, robots, and guides: New tools for neurological rehabilitation" in Winters, P.E. (eds.) Biomechanics and Neural Control of Movement, Berlin, Springer-Verlag, 1999.

[3] Lum, P. S., Reikensmeyer, D. J. et al. "Robotic assist devices for bimanual physical therapy: preliminary experiments" IEEE Transactions on Rehabilitation Engineering Vol. 1: 185-191, 1993. 
[4] Aisen, M. L., Krebs, H. I. et al. " The effect of robotassisted therapy and rehabilitative training on motor recovery following stroke" Arch Neurol 54(4): 443-6, 1997.

[5] Perry, J. C. and Rosen, J. "Design of a 7 Degree-ofFreedom Upper-Limb Powered Exoskeleton", BioRob 2006The first IEEE / RAS EMBS International Conference on Biomedical Robotics and Biomechatronics, Pisa, Italy, February 20-22, 2006

[6] Sanchez, R. J. Liu, J., Rao, S. Shah, P., Smith, R. Rahman, T. Cramer, S. C., Bobrow, J. E. and Reinkensmeyer, D. J. "Automating arm movement training following severe stroke: functional exercises with quantitative feedback in a GravityReduced Environment" IEEE Transactions on Neural Systems and Rehabilitation Engineering, Vol. 14, No. 3, 2006.

[7] Rahman, T., Sample, W., Jayakumar, S. M., King, M., Wee, J.Y., Seliktar, R., Alexander, M., Scavina, M. and Clark, A. "Passive exoskeletons for assisting limb movement", Journal of Rehabilitation Research \& Development, Volume 43, Number 5, Pages 583-590, 2006.

[8] Herder, J. L., Vrijlandt, N., Antonides, T., Cloosterman, M. and Mastenbroek, P. L. "Principle and design of a mobile arm support for people with muscular weakness" Journal of Rehabilitation Research \& Development, Volume 43, Number 5, Pages 591-604, 2006.

[9] Carignan, C., Liszka, M. and Roderick, S. "Design of an Exoskeleton with Scapula Motion for Shoulder Rehabilitation" Proc. IEEE Int. Conf. on Advanced Robotics (ICAR), Seattle, 524-531, 2005.

[10] Yang, G., Ho, H. L., Chen, W., Lin, W., Yeo, S. H. and Mustafa, S. K. "A haptic device wearable on a human arm" IEEE Conference on Robotics, Automation and Mechatronics, Vol: 1, No: 1, 243-247, 2004

[11] Mustafa, S.K., Yang, G., Yeo, S.H. and Lin, W. "Optimal design of a bio-inspired anthropocentric shoulder rehabilitator" Applied Bionics and Biomechanics, Vol: 3, No: 3, 199-208, 2006.

[12] Weihai, C., Quanzhu, C., Jianbin, Z. and Shouqian Y. "Kinematics Control for a 7-DOF Cable-Driven Anthropomorphic Arm", Proceedings of the IEEE/RSJ International Conference on Intelligent Robots and Systems, Beijing, China, 2006.

[13] Zheng, M., Weihai, C., Shouqian, Y. and Xingming, W. "Kinematic Analysis for a 7-DOF Modular Hybrid-Driven Manipulator" Proceedings of the IEEE International Conference on Industrial Informatics, 1363-1368, 2006.

[14] http://www.libertytechnology.com Boston Arm Systems, 2003.

[15 ] Ullrich, N. G., Di Lieto, G., Salsedo, F. and Bergamasco, M. "Design and optimization of a purely rotational 3-DOF haptic device" Proceedings of 10th IEEE International Workshop on Robot and Human Interactive Communication, 2001.

[16] Oh, S. and Agrawal, S. K. "Feasible Workspace of a Set Point Controller for a Cable Suspended Robot with Input
Constraints and Disturbances", IEEE Transactions on Control System Technology, 2006, Vol.14, No.4, 735-742.

[17] Oh, S. and Agrawal, S. K. "Computationally Efficient Feasible Set Points Generation and Control of a Cable Robot", IEEE Transactions on Robotics, 2006, Vol.22, No. 3, 551-558.

[18] Oh, S. and Agrawal, S. K. "Cable Suspended Planar Robots with Redundant Cables: Controllers with Positive Tensions", IEEE Transactions on Robotics, 2005, Vol. 21, No. 3, pp. 457-464. 